\title{
Lack of changes in preterm delivery and stillbirths during COVID-19 lockdown in a European region
}

\author{
Juan Arnaez ${ }^{1,2}$ (1) - Carlos Ochoa-Sangrador ${ }^{3} \cdot$ Sonia Caserío $^{4}$ - Elena Pilar Gutiérrez ${ }^{5}$. Maria del Pilar Jiménez ${ }^{6}$. \\ Leticia Castañón ${ }^{7}$. Marta Benito ${ }^{8}$. Ana Peña ${ }^{9}$. Natalio Hernández ${ }^{10}$. Miryam Hortelano ${ }^{11}$. Susana Schuffelmann ${ }^{12}$. \\ M. Teresa Prada ${ }^{13}$ - Pablo Diego ${ }^{14}$. F. Joaquín Villagómez ${ }^{15}$ - Alfredo Garcia-Alix ${ }^{2,16,17}$
}

Received: 21 December 2020 / Revised: 22 January 2021 / Accepted: 4 February 2021 / Published online: 12 February 2021

(C) The Author(s), under exclusive licence to Springer-Verlag GmbH, DE part of Springer Nature 2021

\begin{abstract}
Preliminary data in Europe have suggested a reduction in prematurity rates during the COVID-19 pandemic, implying that contingency measures could have an impact on prematurity rates. We designed a population-based prevalence proportion study to explore the potential link between national lockdown measures and a change in preterm births and stillbirths. Adjusted multivariate analyses did not show any decrease in preterm proportions during the lockdown period with respect to the whole prelockdown period or to the prelockdown comparison periods (2015-2019): 6.5\% (95\%CI 5.6-7.4), 6.6\% (95\%CI 6.5-6.8), and 6.2\% (95\%CI 5.7-6.7), respectively. Proportions of preterm live births did not change during lockdown when different gestational age categories were analyzed, nor when birthweight categories were considered. No differences in stillbirth rates among the different study periods were found: $0.33 \%$ (95\%CI $0.04-0.61$ ) during the lockdown period vs. $0.34 \%$ (95\%CI 0.22-0.46) during the prelockdown comparison period (2015-2019).

Conclusion: We did not find any link between prematurity and lockdown, nor between stillbirths and lockdown. Collaborative efforts are desirable to gather more data and additional evidence on this global health issue.

What is Known:

- Prematurity is associated with increased risk of morbidity and mortality.

- Contingency measures during the COVID-19 pandemic may have an impact on reducing prematurity rates.

What is New:

- Prematurity and stillbirth rates remained stable in Castilla-y-León, a Spanish region, during COVID-19 lockdown.

- The role of behavioral patterns and sociocultural factors in the prevention of preterm birth as a result of lockdown measures remains a subject for debate.
\end{abstract}

Communicated by Daniele De Luca

Juan Arnaez

juan.arnaez@neurologianeonatal.org

Carlos Ochoa-Sangrador

cochoa@saludcastillayleon.es

Sonia Caserío

scaserioc@saludcastillayleon.es

Elena Pilar Gutiérrez

epgutierrez@saludcastillayleon.es

Maria del Pilar Jiménez

mjimenezsau@saludcastillayleon.es

Leticia Castañón

lcastanon@saludcastillayleon.es

Marta Benito

mbenitogu@saludcastillayleon.es

Ana Peña

apenabu@saludcastillayleon.es

\author{
Natalio Hernández \\ nhernandezg@saludcastillayleon.es \\ Miryam Hortelano \\ mhortelano@saludcastillayleon.es \\ Susana Schuffelmann \\ sschuffelmann@saludcastillayleon.es \\ M. Teresa Prada \\ tprada@saludcastillayleon.es \\ Pablo Diego \\ pdiego@saludcastillayleon.es \\ F. Joaquín Villagómez \\ fjvh19@saludcastillayleon.es \\ Alfredo Garcia-Alix \\ alfredoalix@gmail.com
}

Extended author information available on the last page of the article 
Keywords Prematurity $\cdot$ COVID-19 $\cdot$ Lockdown $\cdot$ Stillbirth $\cdot$ Pandemic $\cdot$ Infant

\section{Introduction}

Prematurity is a complex condition associated with increased risk of morbidity and mortality. The estimated preterm birth rate is 8.7 (6.3-13.3) in Europe, and it remains the leading cause of death in early childhood worldwide [1]. However, very few cases of preterm birth can be prevented using currently available strategies [2].

Three studies in Europe have reported a reduction in prematurity rates during the COVID-19 pandemic, suggesting that contingency measures could have an impact on prematurity rates [3-5]. Whether the behavior of prematurity rates is consistent with and in a similar proportion to what is seen in other parts of Europe is not known. In fact, two single-center studies showed a higher rate of stillbirths [6,7], although this was not subsequently corroborated in a more robust national study in England [8].

Our aim was to explore, in a population-based prevalence proportion study, the potential link between national lockdown measures and changes in preterm births and stillbirths.

\section{Methods}

\section{Setting}

A nationwide lockdown was adopted in Spain on March 15, 2020, in response to the COVID-19 pandemic and was extended to May 3, 2020, with traffic and mobility restrictions; most of the workforce had to adapt to a work-from-home model, and activities and institutions deemed nonessential were shut down. On May 4, 2020, a four-phase deescalation period was implemented which finished on June 21, 2020, with gradually increased mobility and social interaction, as well as the return of most people to their jobs as long as it was not possible for them to work from home.

\section{Study population and data sources}

The study was a population prevalence proportion study conducted in a total area of $94,226 \mathrm{~km}^{2}$ and with $2,408,000$ million people (Castilla-y-León region) served to by 13 hospitals with perinatal care. Retrospective descriptive datasets from January 2015 were linked from the neonatal admission register and the labor ward register. Duplications of records of infants transferred among the hospitals were checked.
Births were categorized according to the gestational age (weeks + days): extremely premature $(23+0-27+6)$, very premature $(28+0-31+6)$, moderate-to-late premature $(32+$ $0-36+6)$, term $(37+0-41+6)$, and late term (after $42+0)$.

Birthweight of infants was categorized as very low (VLBW, <1500 g) and extremely low (ELBW, <1000 g).

No cases were excluded, and other variables, including sex, type of delivery, multiple pregnancies, and cases of death including intrauterine fetal deaths and perinatal deaths (moribund state at birth) above $23+0$ weeks' gestational age, were retrieved.

\section{Statistical analysis}

The clinical research ethics board of the coordinating hospital (University Hospital of Burgos) approved the study with a waiver of informed consent (protocol number 2358).

Joinpoint regression analysis was used to study varying trends over the study period. Changes in the composition of gestational age and birthweight categories between the COVID-19 period and the prelockdown periods were obtained with proportions and their $95 \%$ confidence interval estimations. Multivariate binomial logistic regression models were used adjusting for confounder variables including hospital, sex, type of delivery, and multiple pregnancies.

The analysis was performed using IBM SPSS Statistics V.26. A two-sided $p$ value of less than 0.05 defined statistical significance.

\section{Results}

We included a total of 70,024 births (67,512 singletons) and 68,998 infants $(69,715$ live infants) born from January 1, 2015 , to June 21,2020 . The rate of daily births per year showed a progressive decline of $19.90 \%$ (95\% CI 16.72 23.07) from 2015 to 2020 . There was no time point at which the trend significantly changed.

We identified 4528 premature live births, with a gestational age below $37+0$ weeks $(6.61 \%, 95 \%$ CI $6.42-6.80 \%)$ during the study period. Births were distributed into gestational age categories as shown in Table 1. Adjusted multivariate analysis did not show any decrease in preterm proportions during the COVID-19 period, either during the lockdown or the lockdown-deescalation period, with respect to the whole prelockdown period (OR $0.93,95 \%$ CI $0.75-1.15$ and OR $0.99,95 \%$ CI $0.85-1.15$, respectively) or to the same period 


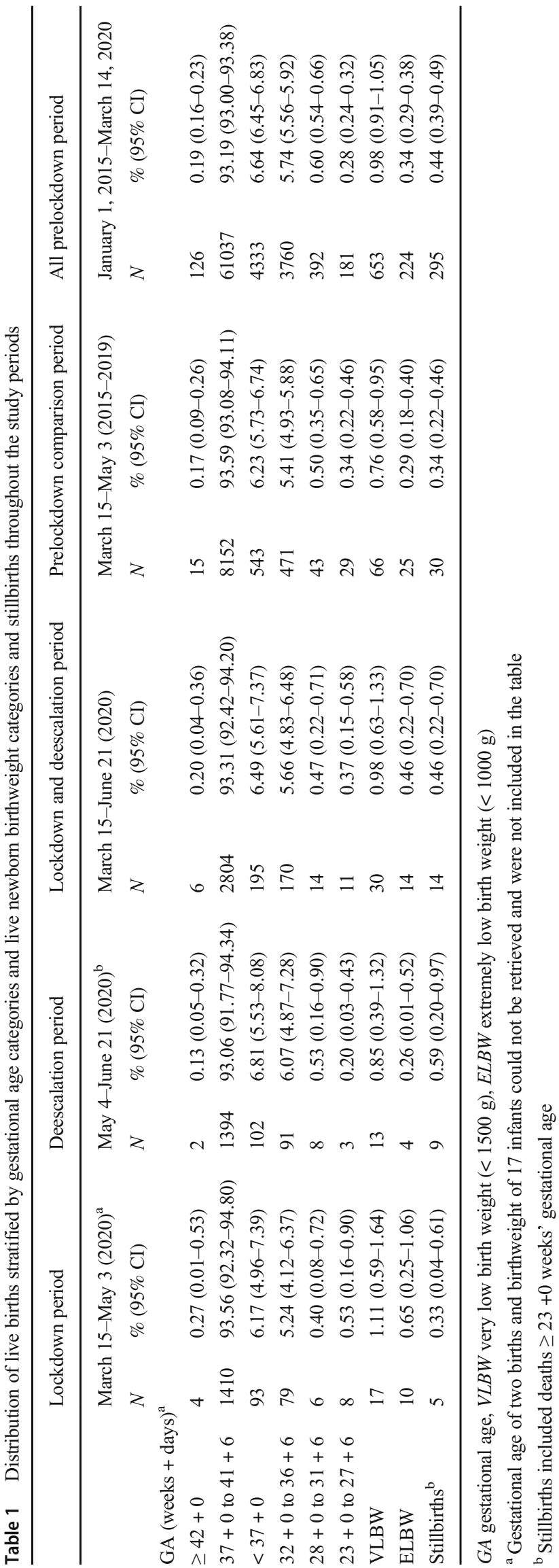

in previous years (OR 0.97, 95\% CI 0.77-1.22 and OR 1.01, 95\% CI 0.86-1.18, respectively). Proportions of preterm live births did not change during lockdown when different gestational age categories were analyzed, except for a slightly significant increase among the extremely premature births $(23+0-27+6$ weeks): OR 2.09 (95\% CI 1.02-4.28; $p=0.042)$. When separate analyses were made within singleton births, no differences were found, nor were there any for extremely premature births: OR 1.38 (95\% CI 0.61-3.12; $p=0.438$ ) (Fig. 1).

As multiple births and type of birth may be associated with preterm birth, we investigated interaction effects and we concluded that despite the fact that there is no increase in multiple births during the lockdown period, multiple births were at greater risk of prematurity during lockdown than in previous periods: $248 / 2451(10.1 \%)$ vs. $12 / 49$ (24.5\%).

Analyses of birthweight categories showed an increase in ELBW among all live newborns during the lockdown period: OR 2.21 (95\% CI 1.16-4.21; $p=0.016$ ), but this was not the case when both the lockdown and the deescalation periods were considered. However, these results faded when only singleton births were considered: OR 1.19 (95\% CI 0.44-3.23; $p$ $=0.724$ ).

A total of 309 stillbirths $(0.44 \%, 95 \%$ CI $0.39-0.49)$ were documented during the study period, five $(0.33 \%, 95 \% \mathrm{CI}$ 0.04-0.61) of them during the lockdown period and nine $(0.59 \%, 95 \%$ CI $0.20-0.97)$ during the deescalation period. Adjusted analysis showed no differences in stillbirth rates, during either the lockdown or lockdown plus the deescalation period, with respect to the whole prelockdown period (OR $0.90,95 \%$ CI $0.37-2.18$ and OR 0.98 , 95\% CI $0.53-1.79$, respectively) or to the same period in previous years (OR $1.22,95 \%$ CI $0.45-3.23$ and OR $1.01,95 \%$ CI $0.57-2.06$, respectively). No differences were found when only singleton stillbirths were considered.

Thirty-five out of 3031 (1.14\%; 0.77-1.52) infants during the lockdown and deescalation period were born from mothers with COVID-19; one of them died in utero at term age. None of the infants had positive results for PCR SARS-CoV-2; 6 infants $(17.1 \%)$ were premature.

\section{Discussion}

On March 15, 2020, mitigation measures to prevent the spread of infection and limit its health effects on the general population were taken by the Spanish government.

Individual observations in perinatal care units developed as a natural experiment into analysis of what might have happened to the prematurity rate during the pandemic. Three national studies, in Denmark [4], Ireland [5], and the Netherlands [3], noted a decrease in the prematurity rate compared to previous periods. Another study conducted at a 
Fig. 1 Preterm birth percentages stratified by gestational age categories twelve weeks before and after the start of lockdown (March 15, 2020) are expressed in weeks + days. The $Y$ axis shows the percentage of premature births per total number of births. The $X$ axis shows the time in weeks before and after the "start week" (March 15 to March 22, 2020)

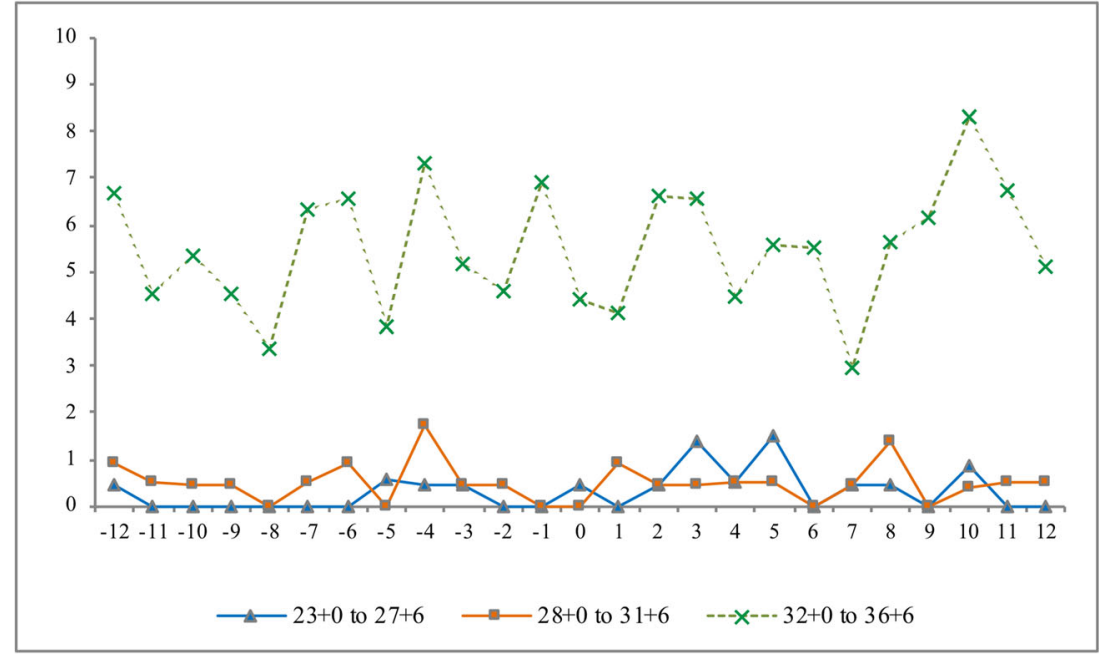

London hospital [6] did not find this decline but showed an increase in stillbirths following the COVID-19 pandemic. This was also found in a single-center retrospective cohort study [7]. However, a most robust study including regional and national data in England found no link between stillbirths and lockdown [8].

Furthermore, the approach to prematurity rate research was not the same in all the studies. In the Irish study, the cohort comprised infants from 22 weeks of gestation stratified into extremely low birthweight and very low birthweight, including multiple gestations, but the period of study commenced in January 1, 2020, rather than March 12, 2020, when lockdown measures were implemented in Ireland. Similarly, a comparison of birth outcomes was made in a London hospital from February 1,2020, but it did not specifically address the effects of the lockdown.

In contrast, the Dutch and Danish studies used national databases containing data on all live singleton babies that underwent neonatal blood spot, and temporal preterm birth patterns during the lockdown period were compared across the same time windows in previous years; singleton births from 24 weeks of gestation were included in the Dutch study, but this was not specified in the Danish study. The evidence of a decline in the prematurity rate also differed between these two studies. While the observed reduction in preterm births in Denmark affected predominantly premature infants $<28$ weeks gestational age during the four weeks of lockdown [4], the decrease in preterm births in the Netherlands was statistically significant only in the $32-36+6$ weeks gestational age group and only after implementation of the March 9 measures and until March 15, but not afterwards [3].

Our study offers more evidence that no link between prematurity and lockdown, nor between stillbirths and lockdown, exists. Our results match those of another recent study that also found no changes during the lockdown period [9]. The explanation for these findings may lie in the differences in the
COVID-19 mitigation measures and the risk factors for prematurity from country to country. Putative potential contributors to the studies that found a lower tendency include increased focus on hygiene and home lockdown, which offered a reduction in work-related stress, greater opportunity for rest/nutritional support, and reduced exposure to infection or air pollution $[4,5]$. Unfortunately, like others, our study is retrospective and lacks investigation of potential mechanisms underlying the association between preterm delivery and lockdown measures.

Our data showed that multiple births were at greater risk of prematurity during the lockdown period. However, due to the low number of preterm infants among multiple births during lockdown, this observation should be approached cautiously. Whether a change in the premature pattern with multiple births may have been influenced by contingency measures remains to be determined in more extensive studies.

In conclusion, the association between stillbirths or the decreased number of premature births and nationwide lockdown remains a subject for debate. Research in this area enables a close look at the role of behavioral patterns and sociocultural factors in the prevention of preterm birth. Collaborative efforts are desirable to gather data and evidence concerning this global health problem.

Abbreviations CI, Confidence interval; ELBW, Extremely low birth weight; IQR , Interquartile range; OR , Odds ratio; SD , Standard deviation; VLBW, Very low birth weight

Author's contributions J.A and A.GA. designed and conceptualized the study, analyzed and interpreted the data, and drafted the manuscript for intellectual content of the paper. C.O. analyzed and interpreted the data and participated in the drafting of the manuscript. The rest of the authors contributed to the acquisition of data and vouched for its accuracy and completeness. All authors made a substantive contribution to revising the manuscript for intellectual content and have approved the final version for publication. 
Data availability The datasets generated during and/or analyzed for the present study are available from the corresponding author on reasonable request.

\section{Declarations}

Ethics approval This is an observational study. The clinical research ethics board of the coordinating hospital confirmed that no ethical approval was required (protocol number 2358).

Conflict of interest The authors declare that they have no conflict of interest.

\section{References}

1. Chawanpaiboon S, Vogel JP, Moller AB, Lumbiganon P, Petzold M, Hogan D, Landoulsi S, Jampathong N, Kongwattanakul K, Laopaiboon M, Lewis C, Rattanakanokchai S, Teng DN, Thinkhamrop J, Watananirun K, Zhang J, Zhou W, Gulmezoglu AM (2019) Global, regional, and national estimates of levels of preterm birth in 2014: a systematic review and modelling analysis. Lancet Glob Health 7(1):e37-e46. https://doi.org/10.1016/S2214109X(18)30451-0

2. Matei A, Saccone G, Vogel JP, Armson AB (2019) Primary and secondary prevention of preterm birth: a review of systematic reviews and ongoing randomized controlled trials. Eur J Obstet Gynecol Reprod Biol 236:224-239. https://doi.org/10.1016/j. ejogrb.2018.12.022

3. Been JV, Burgos Ochoa L, Bertens LCM, Schoenmakers S, Steegers EAP, Reiss IKM (2020) Impact of COVID-19 mitigation measures on the incidence of preterm birth: a national quasi-experimental study. Lancet Public Health 5(11):e604-e611. https://doi.org/10. 1016/S2468-2667(20)30223-1
4. Hedermann G, Hedley PL, Baekvad-Hansen M, Hjalgrim H, Rostgaard K, Poorisrisak P, Breindahl M, Melbye M, Hougaard DM, Christiansen M, Lausten-Thomsen U (2020) Danish premature birth rates during the COVID-19 lockdown. Arch Dis Child Fetal Neonatal Ed 106:93-95. https://doi.org/10.1136/archdischild-2020319990

5. Philip RK, Purtill H, Reidy E, Daly M, Imcha M, McGrath D, O'Connell NH, Dunne CP (2020) Unprecedented reduction in births of very low birthweight (VLBW) and extremely low birthweight (ELBW) infants during the COVID-19 lockdown in Ireland: a 'natural experiment' allowing analysis of data from the prior two decades. BMJ Glob Health 5(9):e003075. https://doi.org/10.1136/ bmjgh-2020-003075

6. Khalil A, von Dadelszen P, Draycott T, Ugwumadu A, O'Brien P, Magee L (2020) Change in the incidence of stillbirth and preterm delivery during the COVID-19 pandemic. JAMA. 324:705. https:// doi.org/10.1001/jama.2020.12746

7. Mor M, Kugler N, Jauniaux E, Betser M, Wiener Y, Cuckle H, Maymon R (2020) Impact of the COVID-19 pandemic on excess perinatal mortality and morbidity in Israel. Am J Perinatol. https:// doi.org/10.1055/s-0040-1721515

8. Stowe J, Smith H, Thurland K, Ramsay ME, Andrews N, Ladhani SN (2020) Stillbirths during the COVID-19 pandemic in England, April-June 2020. JAMA. 325:86-87. https://doi.org/10.1001/jama. 2020.21369

9. Handley SC, Mullin AM, Elovitz MA, Gerson KD, MontoyaWilliams D, Lorch SA, Burris HH (2021) Changes in preterm birth phenotypes and stillbirth at 2 Philadelphia hospitals during the SARS-CoV-2 pandemic, March-June 2020. JAMA 325(1):87-89. https://doi.org/10.1001/jama.2020.20991

Publisher's note Springer Nature remains neutral with regard to jurisdictional claims in published maps and institutional affiliations. 


\section{Affiliations}

Juan Arnaez ${ }^{1,2}$ - Carlos Ochoa-Sangrador ${ }^{3}$. Sonia Caserío ${ }^{4}$ - Elena Pilar Gutiérrez ${ }^{5}$. Maria del Pilar Jiménez ${ }^{6}$. Leticia Castañón ${ }^{7}$. Marta Benito ${ }^{8} \cdot$ Ana Peña $^{9} \cdot$ Natalio Hernández $^{10} \cdot$ Miryam Hortelano $^{11}$. Susana Schuffelmann ${ }^{12}$. M. Teresa Prada ${ }^{13} \cdot$ Pablo Diego $^{14} \cdot$ F. Joaquín Villagómez ${ }^{15} \cdot$ Alfredo Garcia-Alix $^{2,16,17}$

1 Departamento de Pediatría (Neonatología), Complejo Asistencial Universitario de Burgos, Burgos, Spain

2 NeNe Foundation, Madrid, Spain

3 Departamento de Pediatría, Complejo Asistencial de Zamora, Zamora, Spain

4 Departamento de Pediatría (Neonatología), Hospital Universitario Rio Hortega de Valladolid, Valladolid, Spain

5 Departamento de Pediatría (Neonatología), Complejo Asistencial Universitario de Salamanca, Salamanca, Spain

6 Departamento de Pediatría (Neonatología), Complejo Asistencial de Ávila, Ávila, Spain

7 Departamento de Pediatría (Neonatología), Complejo Asistencial Universitario de León, León, Spain

8 Departamento de Pediatría (Neonatología), Hospital Clínico Universitario de Valladolid, Valladolid, Spain
9 Departamento de Pediatría, Complejo Asistencial de Soria, Soria, Spain

10 Departamento de Pediatría (Neonatología), Complejo Asistencial de Zamora, Zamora, Spain

11 Departamento de Pediatría (Neonatología), Complejo Asistencial de Segovia, Segovia, Spain

12 Departamento de Pediatría, Hospital Santos Reyes, Aranda de Duero, Spain

13 Departamento de Pediatría, Hospital El Bierzo, Ponferrada, Spain

14 Departamento de Pediatría, Hospital Santiago Apóstol, Miranda de Ebro, Spain

15 Departamento de Pediatría, Complejo Asistencial de Palencia, Palencia, Spain

16 Institut de Recerca Pediátrica, Hospital Sant Joan de Déu, Barcelona, Spain

17 University of Barcelona, Barcelona, Spain 